\title{
Riesgo ocupacional y autocuidado en enfermería, en atención primaria de salud
}

\section{Occupational risk and self-care in nursing, in APS- AZUAY 2019}

\author{
Tania Carolina Pérez López ${ }^{* 1}$, Liliana Elizabeth Sotamba Aucapiña ${ }^{1}$, Nataly Quito Peralta ${ }^{1}$, Cristina Urgilés \\ Barahona $^{1,2}$, Fabricio Israel Febres Calderón 1 \\ ${ }^{1}$ Universidad Católica de Cuenca, Ecuador \\ ${ }^{2}$ Universidad Técnica Particular de Loja, Ecuador \\ *tcperezl26@est.ucacue.edu.ec
}

DOI: https://doi.org/10.26871/killkanasalud.v4i4.745

\begin{abstract}
Resumen
Objetivo: El presente estudio tiene el fin de identificar los riesgos ocupacionales y la relación con el autocuidado en los profesionales de enfermería que laboran en el primer nivel de atención en los Distritos 01D01 y 01D02 de la provincia del Azuay en 2019. Metodología: Esta investigación tiene enfoque cuantitativo, correlacional de corte transversal. Para la misma se ha seleccionado una población de estudio que estuvo constituida por profesionales enfermeras/os que laboran en los distritos de salud 01D01 y 01D02. Para determinar los objetivos del estudio, se utilizaron dos instrumentos que fueron: escala de percepción de riesgo con alfa de Cronbach de 0,892 y agencia de autocuidado con 0,81. Resultados: Los resultados del presente estudio se analizaron con el programa SPSS V15. Donde el $60.6 \%$ pertenece al grupo etario de 20-35 años de edad, estado civil casado 66,5\%, experiencia laboral de 1-10 años 60\%, contratación ocasional 83,2\%, el 45,2\% sufrió algún tipo de accidente laboral, con percepción de riesgo media en el $40 \%$, conducta preventiva normal $41,3 \%$ y una capacidad media de autocuidado $79,4 \%$. Se ha identificado también que los riesgos mayormente percibidos por los enfermeros/as fueron: el trabajo con productos biológicos y el trabajo con elementos e instrumentación punzante y/o cortante $100 \%$, elementos comúnmente utilizados durante su jornada laboral. Conclusiones: Durante el desarrollo del estudio se logró evidenciar que no existe relación entre las variables de percepción de riesgo laboral y autocuidado en los profesionales de enfermería, esto por una mejor aplicación de diversas medidas de seguridad laboral, implementadas de forma personal e institucional.
\end{abstract}

Palabras clave: enfermería del trabajo, riesgos laborales, autocuidado, atención primaria de salud, salud pública .

\section{Abstract}

Objective: This study aims to identify occupational risks and the relationship with self-care in nursing professionals working at the first level of care in Districts 01D01 and 01D02 of the province of Azuay in 2019. Methodology: This research has a quantitative, correlational cross-sectional approach. A study population has been selected for this study, which was constituted by nursing professionals who work in health districts $01 D 01$ and 01D02. To determine the objectives of the study, two instruments were used: risk perception scale with Cronbach's alpha of 0.892 and self-care agency with 0.81. Results: The results of the present study were analyzed with the SPSS V15 program. Where $60.6 \%$ belong to the age group 20-35 years, married marital status 66.5\%, work experience 1-10 years $60 \%$, occasional hiring $83.2 \%$, 45.2\% suffered some type of occupational accident, with average risk perception in $40 \%$, normal preventive behavior $41.3 \%$ and average self-care capacity $79.4 \%$. It has also been identified that the risks mostly perceived by nurses were: working with biological products and working with piercing and/or cutting elements and instruments $100 \%$, elements commonly used during their working day. Conclusions: During the development of the study, it became evident that there is no relationship between the variables of perception of occupational risk and self-care in nursing professionals. This is due to the improved application

Keywords: occupational nursing, occupational risks, self care, primary health care, public health .

\section{Introducción}

Los riesgos laborales son situaciones que se presentan en el lugar de trabajo del individuo, exponiendo al empleado a una mayor exposición. Los profesionales de enfermería dentro de su ambiente laboral se encuentran expuestos a sufrir de riesgos ocupacionales de diferente naturaleza; tomando en consideración que el riesgo es toda aquella situación que puede ocasionar un daño. ${ }^{1}$ 
La salud ocupacional estudia las condiciones del trabajo y el impacto de la salud de los trabajadores; el área laboral en el que se desempeña el personal sanitario, entre ellos, el personal de enfermería se encuentran continuamente expuestos a sufrir accidentes y enfermedades profesionales que pueden provocar repercusiones no solamente económicas, sino también afectar el bienestar físico y emocional a corto y largo plazo.

Buscó evidenciar los riesgos a los que se exponen los profesionales de enfermería y cuál es la conducta de autocuidado que poseen, con el propósito de conocer el grado de relación de estas dos variables, que dentro del ámbito de salud guardan gran relevancia; debido que el enfermero debe vigilar por su bienestar y encontrarse en condiciones óptimas de salud para preservar la salud del individuo a su cuidado.

Esta investigación es trascendental por que identifica los riesgos a los que está expuesto el profesional, haciendo que estos no pasen desapercibidos al momento del desempeño, por lo que es fundamental detectar a tiempo la presencia de los causantes que podrían producir déficit en la salud con el fin de evitar enfermedades profesionales.

\section{Marco teórico y antecedentes}

La OMS en noviembre del 2017 calculó que anualmente 12,2 millones de personas sufre accidentes la Organización Internacional del Trabajo (OIT) informó que a causa de accidentes laborales o enfermedades relacionadas al trabajo, mueren cada año 317 millones de personas víctimas de accidentes, demostrando que existe la carga económica de las malas prácticas de seguridad y salud. ${ }^{2}$

La Organización Panamericana de la Salud -OPS- ${ }^{3}$ indica que se presentan aproximadamente treinta y seis lesiones relacionadas con el trabajo por minuto y 300 trabajadores fallecen a diario a causa de accidentes ocupacionales, cada año casi 800 muertes alrededor del mundo se deben a lesiones ocupacionales y 11.000 a enfermedades ocupacionales. Los profesionales enfermeros son una población vulnerable para padecer riesgos en su trabajo, ya que se encuentran diariamente propensos a adquirir diferentes riesgos laborales, tomando en cuenta que un riesgo laboral es la probabilidad de que se produzca un deterioro o contratiempo en el medio laboral. ${ }^{4}$

La Atención Primaria de Salud (APS) conforma el primer nivel de contacto entre el individuo, familia y comunidad con el Sistema Nacional de Salud (SNS) en donde la promoción y la prevención de la salud son acciones propias de enfermería, dentro de sus funciones el cuidado de la salud, tanto a población sana como enferma, ${ }^{4} \mathrm{El}$ riesgo a sufrir accidentes laborales puede afectar su desempeño diario existe el contacto directo y continuo con el paciente, motivo por el cual presentan el índice más alto de enfermedades profesionales y accidentes laborales debido a su desempeño, ${ }^{6}$

\subsection{Salud ocupacional}

Actividades dirigidas hacia la promoción de la calidad de vida de los trabajadores, diagnóstico precoz, tratamiento oportuno, rehabilitación, readaptación laboral, ${ }^{7}$

La entidad estatal que regula, vigila, planifica, coordina, controla y gestiona el proceso de Salud del Ecuador es el Ministerio de Salud Pública (MSP), el mismo que se encarga de cuidar del personal de atención al usuario. ${ }^{10}$

\subsection{Atención Primaria De Salud (APS)}

La APS en una conferencia brindada por la OMS fue definida como: "La asistencia esencial, basada en métodos y tecnologías prácticas, científicamente fundadas y socialmente aceptables, puesta al alcance de todos los individuos, familias y comunidades; a un costo que la comunidad y el país puedan soportar, en todas y cada una de las etapas de su desarrollo"9".

Dentro del SNS (Sistema Nacional de Salud), representa el núcleo principal y por consiguiente el primer nivel de contacto con el individuo, familias y comunidades. ${ }^{8,9}$

El Sistema de Salud Ecuatoriano a nivel urbano cuenta con un médico, una enfermera y un técnico de salud primario (TAPS) por cada 4000 habitantes, en tanto que en el ámbito rural existe un médico, una enfermera y un TAPS por 1500 a 2500 habitantes, ${ }^{10}$ por lo tanto las poblaciones asignadas a la atención primaria varían según la disponibilidad geográfica y la distribución de la población. ${ }^{11}$

\subsection{Medio ambiente y trabajo}

El Medio ambiente es considerado "Un conjunto de factores de orden físico, químico y biológico que actúan sobre el ser humano, ya que brindan recursos necesarios para la supervivencia; mientras que el entorno laboral es el conjunto de condiciones que rodean a la persona de forma directa o indirecta, influyendo en su salud y calidad de vida", 7 puesto que se relacionan con factores físicos, administrativos y sociales que afectan el ambiente laboral causando cambios en el estado de salud, ${ }^{16}$ ya que generan riesgos que pueden ser de origen tanto biológico, ergonómico, químico, físico y psicosocial. ${ }^{7}$

\section{Bioseguridad}

La bioseguridad hace referencia a las medidas y normas preventivas, destinadas a mantener un control de los factores de riesgos laborales, para lograr la prevención frente a riesgos propios de la actividad diaria, para que el desarrollo o producto final de dichos procedimientos no atenten contra la seguridad de los trabajadores de la salud, ${ }^{12}$ se necesita de reglas y prohibiciones enlazadas con el reconocimiento del ambiente de trabajo; por lo cual, a continuación se detallan las medidas preventivas. ${ }^{13,14}$ 
Los profesionales de la salud deben de contar con un esquema de vacunación con la finalidad de evitar enfermedades o infecciones. Las inmunizaciones que son recomendadas por tener un aporte significativo son contra la hepatitis B (HB), fiebre amarilla (FA), Sarampión y Rubéola (SR), difteria y tétanos (dT) e influenza, ${ }^{15}$ proporcionar seguridad al paciente $\mathrm{y}$, sobre todo prevenir, diferentes riesgos producidos durante las tareas diarias. ${ }^{12,14}$

Los riesgos ocupacionales en los profesionales de enfermería pueden ser riesgos de origen biológico, físico, ergonómico, psicosocial y químico. 5,16

\subsection{Leyes de seguridad y salud ocupacional}

Existen leyes que amparan a los empleadores en caso de sufrir accidentes o riesgos laborales por lo que es relevante mencionar las políticas vigentes en relación con la salud y seguridad ocupacional en el Ecuador. ${ }^{17}$ A pesar de que existen leyes que vigilan el bienestar del trabajador, los profesionales de enfermería son los que mayor riesgo tienen de adquirir algún accidente o enfermedad, ya que cumplen un papel fundamental en los servicios de la Atención Primaria; de la misma manera, las malas condiciones del ambiente de trabajo también influyen a que los profesionales se expongan a riesgos ocupacionales al no contar con un ambiente laboral seguro y adecuado durante la realización de sus actividades diarias. .,16,17 $^{2}$

\subsection{Teorías de enfermería}

La enfermería como arte y ciencia; puso énfasis en la importancia de la interrelación entre el individuo y el medio ambiente. ${ }^{20}$ Para Nightingale, el entorno en el que trabaja el individuo es de importancia crucial porque todas las condiciones e influencias externas pueden afectar la vida y el desarrollo de una persona y como resultado de esta ocurrencia de enfermedades o accidentes. ${ }^{18,19}$

\subsection{Conceptos metaparadigmáticos de Dorothea Orem}

Persona: representa al hombre como un organismo biológico, racional y pensante. Como un todo dinámico integrado con la capacidad de conocerse, usar ideas, palabras y símbolos para pensar, comunicarse y guiar esfuerzos, la capacidad de reflexionar sobre su propia experiencia y eventos de seguridad para implementar medidas de autocuidado dependientes. ${ }^{18,20}$

Salud: estado de la persona que condiciona sus necesidades de atención general, habilidades y capacidades para satisfacerlas y concibe la atención como necesidades que todas las personas tienen a lo largo de sus vidas y que contribuirán a su salud, bienestar y desarrollo. ${ }^{20}$

Enfermería: servicio humano, que se proporciona cuando una persona no puede cuidarse a sí misma para mantener la salud, la vida y el bienestar, esto significa proporcionar a las personas o grupos asistencia directa en el autocuidado, de acuerdo con sus requisitos, debido a las deficiencias que se dan por sus situaciones personales. $^{20}$
Entorno: conjunto de elementos del medio donde se encuentra la persona, que pueden ser utilizados para la realización de cuidados que contribuyan a la salud y bienestar del individuo. ${ }^{18,20}$

\subsection{Autocuidado}

El concepto de autocuidado de Dorothea E. Orem se anunció en 1969, su teoría se basa principalmente en la atención brindada por y para sí mismo. También lo considera una función reguladora que tiene la persona y que debe realizar conscientemente por sí misma para mantener su vida, salud, desarrollo y bienestar. ${ }^{4,18,21}$ Dorothea E. Orem señaló que se requieren tres fases para implementar su idea de autocuidado:

- Tener en cuenta los requisitos de la acción.

- Toma de decisiones, debe ser capaz de pensar sobre la relación causa-efecto.

- Actuar, que requiere estar motivado para hacerlo, ser capaz de persistir en el curso de la acción y ser físicamente capaz de llevarlo a cabo. ${ }^{18}$

\subsection{Agencia de autocuidado}

Son las capacidades específicas que posee el individuo para cumplir con las tareas de su propio cuidado. ${ }^{4}$ Es decir, se refiere a la "Totalidad de actividades que un individuo inicia para mantener la vida y desarrollarse de una forma que sea normal para él".22

\subsection{Déficit de autocuidado}

Esta teoría establece que las personas están sujetas a limitaciones relacionadas o desviadas de su salud, lo que las hace incapaces de autocuidado continuo o lo hacen ineficaz o incompleto Con lo anteriormente expuesto, se considera relevante que los accidentes ocupacionales son parte inherente del desempeño diario de los trabajadores, tomando en cuenta que en el entorno en donde laboran los profesionales de enfermería se presentan riesgos de diferente índole, por lo que el profesional debe tomar medidas preventivas vinculadas con el autocuidado para asegurar la salud. ${ }^{21,23}$

\section{Materiales y métodos}

Estudio con enfoque cuantitativo, de alcance correlacional, de corte transversal. La población de estudio estuvo conformada por los enfermeros pertenecientes a los

Distritos de salud 01D01 y 01D02 de la provincia del Azuay. El Distrito 01D01 está constituido por 82 profesionales de enfermería y el Distrito 01 D02 por 103 profesionales, lo cual abarca una suma total de 185 profesionales enfermeros/as. Se incluyó a profesionales de enfermería que acepten ser parte de la investigación y firmen el consentimiento informado, profesionales de enfermería que se encuentren laborando con cualquier tipo de contrato laboral. Se excluyeron del estudio al profesional de enfermería que se encuentre en periodo de licencia por maternidad, vacaciones, calamidad doméstica o algún motivo de fuerza 
mayor que le impidan estar en su área de trabajo y al profesional de enfermería que a pesar de firmar el consentimiento informado deciden no participar en la investigación.

Para el estudio de los riesgos ocupacionales se empleó un cuestionario de 19 ítems, el mismo que dispone es su primera parte la ausencia o presencia del riesgo en donde el profesional de Enfermería indicará el mismo según su percepción y lugar de trabajo. Posteriormente hay tres subescalas que miden: grado de existencia del riesgo, efectos para la salud y control de efectos negativos, Estas subescalas, tienen opciones de respuesta tipo likert, desde 1 a 7 que corresponden: 1: nunca, 2, casi nunca, 3 pocas veces, 4 algunas veces, 5 frecuentemente, 6 casi siempre, 7 siempre.

Para la variable dependiente de Autocuidado se utilizó la escala de agencia de autocuidado, compuesta por 24 preguntas con formato de respuesta likert. La posible respuesta a cada una de las preguntas es: 1: Nunca, 2: casi nunca, 3: casi siempre y 4: siempre, clasificadas según el puntaje obtenido como baja capacidad de agencia de autocuidado (menor a 59 puntos) o alta capacidad de agencia de autocuidado (60 a 96 puntos). Las preguntas 6, 11 y 20, deben revertirse ya que están con enunciados negativos. ${ }^{4}$

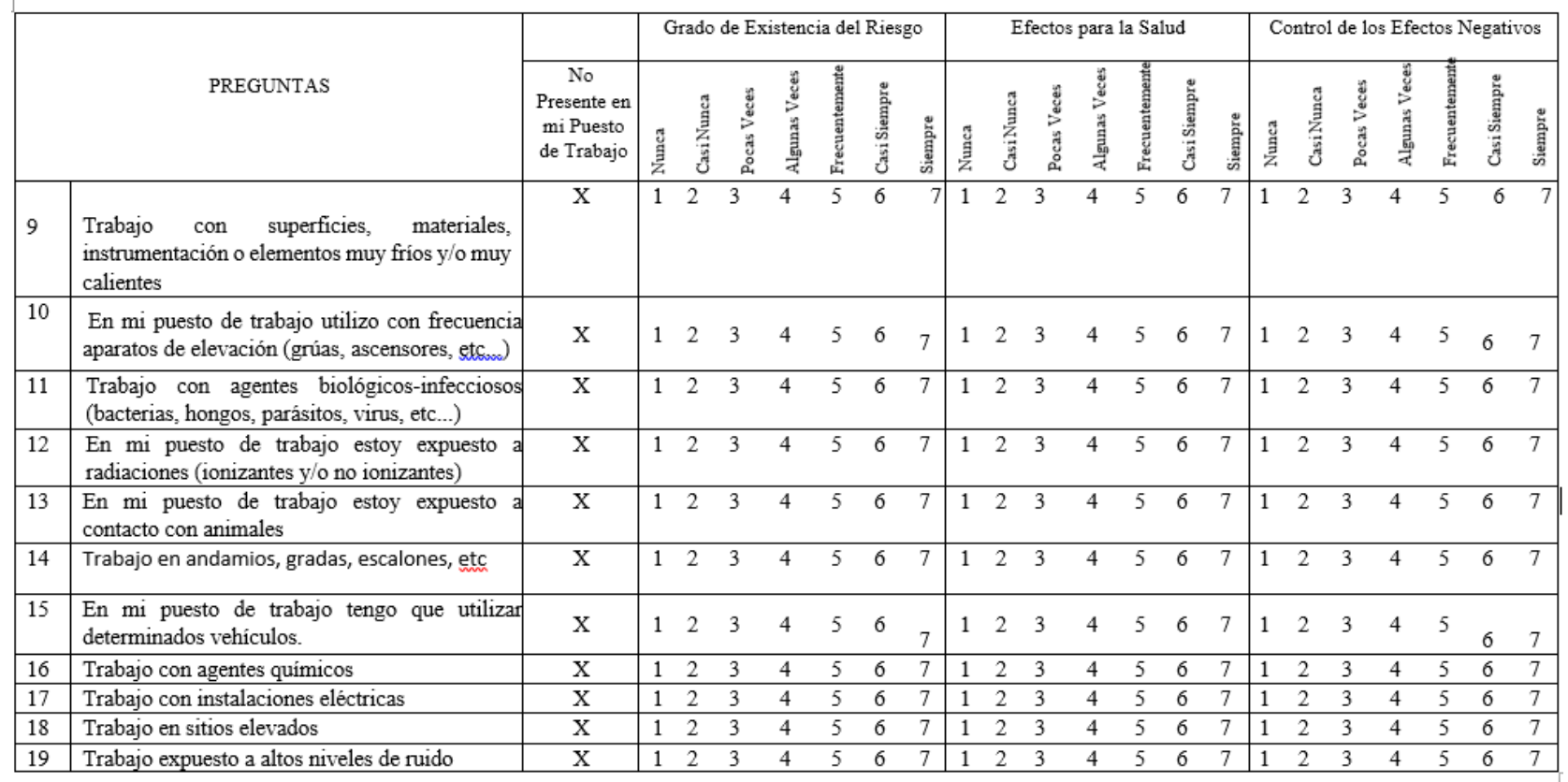

Si usted considera que hay algún riesgo en su trabajo que no haya sido mencionado, indíquelo aquí

Fig. 1: Cuestionario de percepción de riesgo laboral

Los datos obtenidos en este estudio de investigación se ingresaron en una base de datos en Excel 2015 y por consiguiente las medidas estadísticas que se empleo es el programa estadístico SPSS V15. Para el análisis de los datos obtenidos se empleó la estadística descriptiva, las variables cualitativas se analizan en base a medidas de frecuencia y porcentaje, para establecer la asociación entre riesgo laboral y autocuidado se aplicó Odds Ratio con sus respectivos intervalos de confianza y como medida de significancia estadística Chi Cuadrado con un valor p:<0,05; para establecer la diferencia en la percepción de riesgo entre la población de los distritos de salud se aplicó U de Mann Whitney con un p:<0,05, la presentación de los resultados se realiza en tablas. 


\section{ESCALA DE PERCEPCIONN DE RIESGO.}

Instrucciones:

A continuación, se le presentarán una serie de enunciados y usted tendrá que evaluar si está de acuerdo con ellos o no y en qué grado. Para ello deberá rodear con un círculo el número que considere oportuno. Tenga en cuenta que:

\begin{tabular}{|c|c|c|c|c|c|c|}
\hline NUNCA & $\begin{array}{c}\text { CASI } \\
\text { NUNCA }\end{array}$ & $\begin{array}{c}\text { POCAS } \\
\text { VECES }\end{array}$ & $\begin{array}{c}\text { ALGUNAS } \\
\text { VECES }\end{array}$ & FRECUENTEMENTE & $\begin{array}{c}\text { CASI } \\
\text { SIEMPRE }\end{array}$ & SIEMPRE \\
\hline 1 & 2 & 3 & 4 & 5 & 6 & 7 \\
\hline
\end{tabular}

En cada uno de los enunciados aparecen tres tipos de respuesta:

1. - La condición laboral existe en su puesto de trabajo y en qué grado

2.- Si esa condición laboral es perjudicial para su salud y en qué grado

3.- En caso de que sea perjudicial para su salud, si puede controlar sus efectos negativos y en qué grado

Si alguna de las condiciones laborales no existe en su 'trabajo, rodee la X de la opción "No presente en mi trabajo" y pase al siguiente enunciado.

Los resultados de este cuestionario serán utilizados únicamente con fines de investigación, por lo que le rogamos que conteste todas las preguntas con la mayor sinceridad.

Fig. 2: Escala de percepción de riesgo laboral

\begin{tabular}{|c|c|c|c|c|}
\hline ITEMS & Nunca & $\begin{array}{c}\text { Casi } \\
\text { nunca }\end{array}$ & $\begin{array}{c}\text { Casi } \\
\text { siempre }\end{array}$ & Siempre \\
\hline $\begin{array}{l}\text { 1. A medida que cambian las circunstancias voy haciendo } \\
\text { ajustes para mantenerme con salud }\end{array}$ & 1 & 2 & 3 & 4 \\
\hline $\begin{array}{l}\text { 2. Reviso si son buenas las formas que practico habitualmente } \\
\text { para mantenerme con salud. }\end{array}$ & 1 & 2 & 3 & 4 \\
\hline $\begin{array}{l}\text { 3. Si tengo problemas para moverme o desplazarme me las } \\
\text { arreglo para conseguir ayuda. }\end{array}$ & 1 & 2 & 3 & 4 \\
\hline $\begin{array}{l}\text { 4. Puedo hacer lo necesario para mantener limpio el ambiente en } \\
\text { el que vivo. }\end{array}$ & 1 & 2 & 3 & 4 \\
\hline $\begin{array}{l}\text { 5. Hago en primer lugar lo que sea necesario para mantenerme } \\
\text { con salud }\end{array}$ & 1 & 2 & 3 & 4 \\
\hline 6. Me faltan las fuerzas necesarias para cuidarme como debo & 1 & 2 & 3 & 4 \\
\hline 7. Puedo buscar mejores formas de cuidar mi salud de las que tengo ahora & 1 & 2 & 3 & 4 \\
\hline $\begin{array}{l}\text { 8. Cambio la frecuencia con la que me baño para mantenerme } \\
\text { Limpio }\end{array}$ & 1 & 2 & 3 & 4 \\
\hline $\begin{array}{l}\text { 9. Para mantener el peso que me corresponde hago cambios en mis } \\
\text { hábitos alimenticios }\end{array}$ & 1 & 2 & 3 & 4 \\
\hline $\begin{array}{l}\text { 10. Cuando hay situaciones que me afectan, las manejo de } \\
\text { manera que pueda mantener mi forma de ser }\end{array}$ & 1 & 2 & 3 & 4 \\
\hline $\begin{array}{l}\text { 11.Pienso en hacer ejercicio y descansar un poco durante el día, pero no } \\
\text { lleqo a hacerlo }\end{array}$ & 1 & 2 & 3 & 4 \\
\hline $\begin{array}{l}\text { 12. Cuando necesito ayuda puedo recurrir a mis amigos de } \\
\text { Siempre }\end{array}$ & 1 & 2 & 3 & 4 \\
\hline 13. Puedo dormir lo suficiente como para sentirme descansado & 1 & 2 & 3 & 4 \\
\hline $\begin{array}{l}\text { 14. Cuando tengo información sobre mi salud pido explicaciones } \\
\text { sobre lo que no entiendo }\end{array}$ & 1 & 2 & 3 & 4 \\
\hline 15. Examino mi cuerpo para saber si hay algún cambio & 1 & 2 & 3 & 4 \\
\hline $\begin{array}{l}\text { 16. He sido capaz de cambiar hábitos que tenía muy arraigados con tal de } \\
\text { mejorar mi salud. }\end{array}$ & 1 & 2 & 3 & 4 \\
\hline $\begin{array}{l}\text { 17. Cuando tengo que tomar una nueva medicina cuento con una } \\
\text { persona que me brinda información sobre los efectos } \\
\text { secundarios. }\end{array}$ & 1 & 2 & 3 & 4 \\
\hline $\begin{array}{l}\text { 18. Soy capaz de tomar medidas para garantizar que mi familia y } \\
\text { yo no corramos peligro }\end{array}$ & 1 & 2 & 3 & 4 \\
\hline $\begin{array}{l}\text { 19. Soy capaz de evaluar qué tanto me sirve lo que hago para } \\
\text { mantenerme con salud }\end{array}$ & 1 & 2 & 3 & 4 \\
\hline $\begin{array}{l}\text { 20. Debido a mis ocupaciones diarias me resulta difícil sacar } \\
\text { tiempo para cuidarme }\end{array}$ & 1 & 2 & 3 & 4 \\
\hline $\begin{array}{l}\text { 21. Si mi salud se ve afectada yo puedo conseguir la información } \\
\text { necesaria sobre qué hacer }\end{array}$ & 1 & 2 & 3 & 4 \\
\hline 22. Si no puedo cuidarme puedo buscar ayuda & 1 & 2 & 3 & 4 \\
\hline 23. Puedo disponer de tiempo para mi & 1 & 2 & 3 & 4 \\
\hline $\begin{array}{l}\text { 24. A pesar de las limitaciones para movilizarme, soy capaz de } \\
\text { cuidarme como a mí me gusta }\end{array}$ & 1 & 2 & 3 & 4 \\
\hline
\end{tabular}

Fig. 3: Cuestionario de agencia de autocuidado 


\section{Resultados}

El estudio se realizó a la población de profesionales enfermeros pertenecientes a los Distritos de salud 01D01 y 01D02 del Azuay, la población inicial estuvo conformada por 185 profesionales, pero debido a diversas situaciones como: la negatividad a la participación, licencia de maternidad, vacaciones entre otros; únicamente se trabajó con 155 profesionales de enfermería de los cuales los resultados obtenidos se evidencian en las siguientes tablas.

Tabla 1: Distribución de la población según variables sociodemográficas

\begin{tabular}{lcc}
\hline Variable & Frecuencia N & Porcentaje \% \\
\hline \multicolumn{3}{c}{ Edad } \\
\hline $20-35$ años & 94 & 60,6 \\
$36-45$ años & 41 & 26,5 \\
$46-55$ años & 18 & 11,6 \\
$56-65$ años & 2 & 1,3 \\
\hline \multicolumn{3}{c}{ Sexo } \\
\hline Femenino & 149 & 96,1 \\
Masculino & 6 & 3,9 \\
\hline \multicolumn{3}{c}{ Estado civil } \\
\hline Soltero & 43 & 27,7 \\
Casado & 103 & 66,5 \\
Divorciado & 8 & 5,2 \\
Viudo & 1 & 0,6 \\
\hline TOTAL GLOBAL & $\mathbf{1 5 5}$ & $\mathbf{1 0 0} \%$ \\
\hline
\end{tabular}

La tabla 1 muestra que el rango etario que prevalece es el de 20 a 35 años en más de la mitad de la población al igual que el estado civil casado y predomina el sexo femenino $(96,1 \%)$. La tabla 1 muestra que el rango etario que prevalece es el de 20 a 35 años en más de la mitad de la población al igual que el estado civil casado y predomina el sexo femenino $(96,1 \%)$.

Tabla 2: Categorización de agencia de autocuidado

\begin{tabular}{lcc}
\hline Variable & Frecuencia N & Porcentaje \% \\
\hline \multicolumn{3}{c}{ Autocuidado agrupado } \\
\hline Baja capacidad & 12 & 7,7 \\
Mediana capacidad & 123 & 79,4 \\
Alta capacidad & 20 & 12,9 \\
\hline TOTAL & $\mathbf{1 5 5}$ & $\mathbf{1 0 0} \%$ \\
\hline
\end{tabular}

De los 155 profesionales de enfermería encuestados el $79,4 \%$ representan una mediana capacidad de autocuidado en tanto que el $7,7 \%$ se puede evidenciar una baja capacidad de autocuidado.

Tabla 3: Categorización de la percepción de riesgo de los Profesionales de Enfermería

\begin{tabular}{lcc}
\hline Variable & Frecuencia N & Porcentaje \% \\
\hline \multicolumn{3}{c}{ Percepción de riesgo agrupada } \\
\hline Baja percepción & 47 & 30,3 \\
Mediana percepción & 62 & 40 \\
Alta percepción & 46 & 29,7 \\
\hline TOTAL & $\mathbf{1 5 5}$ & $\mathbf{1 0 0 \%}$ \\
\hline
\end{tabular}

En la tabla 4 se puede evidenciar que prevalece una mediana percepción de riesgo $(40 \%)$, en tanto que, la alta percepción se encuentra en tan solo un $29,7 \%$ de la población.

Tabla 4: Categorización de la percepción de riesgo de los Profesionales de Enfermería

\begin{tabular}{cccccc}
\hline Variable & $\begin{array}{c}\text { Baja Ca- } \\
\text { pacidad }\end{array}$ & $\begin{array}{c}\text { Alta Ca- } \\
\text { pacidad }\end{array}$ & OR & IC & P \\
\hline \multicolumn{5}{c}{ Percepción de riesgo } \\
\hline $\begin{array}{c}\text { Bajo } \\
\text { Riesgo } \\
\text { Alto } \\
\text { Riesgo }\end{array}$ & $2(1,3 \%)$ & $76(49 \%)$ & 0,649 & $0,105-3,3997$ & 0,639 \\
\hline TOTAL & $\mathbf{1 5 5}$ & $\mathbf{1 0 0} \%$ & & \\
\hline
\end{tabular}

Al aplicar los estadísticos de estimación de autocuidado con la percepción de riesgo se puede observar que no existe una asociación significativa (p 0,639).

\section{Discusión}

El presente estudio, se aplicó en la población de profesionales enfermeros pertenecientes a los distritos de salud 01D01 y 01D02 del Azuay, en quienes se aplicó una escala de percepción de riesgos y de autocuidado, recalcando que la participación era voluntaria y la información recolectada quedará únicamente entre las investigadoras, sin embargo se debe mencionar que hubo el $16 \%$ de pérdidas de la población en estudio, debido a diversos factores como: la negatividad a la participación, personal con licencia de maternidad, vacaciones entre otros.

La población mayoritaria en este estudio está representada por el género femenino con un $96,1 \%$ estos resultados son similares al estudio de Girão-Arcanjo et al, en el año 2018 en donde se encontró que el sexo predominante es el de mujeres con un $92 \%,{ }^{24}$ por otra parte, en la investigación de Espinoza-Benavente también predomina este sexo con un $82 \%,{ }^{4}$ por lo que cabe recalcar que estos porcentajes se les atribuye a este sexo, ya que históricamente la profesión de enfermería a sus inicios era propia del género femenino.

En la investigación se observó que los rangos de edad más predominantes están entre los 20 a 35 años demostrando que existe una población relativamente joven desempeñándose en la APS, lo cual difiere con los resultados del estudio realizado por Pires de Pires et al, en el año 2016 que busca identificar las cargas de trabajo de enfermería en donde obtuvo como resultado que el $61 \%$ de edades estaban comprendidas entre los 30 a 39 años de edad, ${ }^{25}$ dato que resulta superior al de esta investigación. 
Se observa también en esta investigación, que la mayor parte de los profesionales de Enfermería, percibe los riesgos medianamente $(40 \%)$ resultado que tuvo similitud al encontrado en el estudio de Espinoza-Benavente que indica que el $42,9 \%$ de la población en estudio mantiene una percepción laboral de riesgo media. ${ }^{4}$

Una investigación manifiesta que el reconocer los riesgos a los que está expuesto, hace que los individuos tengan comportamientos más preventivos ${ }^{4}$ es así, que en este estudio tan solo el $29 \%$ de los profesionales poseen una conducta preventiva, mientras que el $29,7 \%$ presenta una conducta arriesgada y un $41,3 \%$ presenta una conducta normal.

Se indagó también sobre la conducta de autocuidado en los profesionales enfermeros, considerando que esta forma parte de la vida diaria es así que, en este estudio se observó que tan solo un $12,9 \%$ de los profesionales de enfermería presentan una alta capacidad de autocuidado, mientras que el 74,9\% una mediana agencia de autocuidado y el 7,7\% restante representan una baja agencia a comparación con la investigación de Sánchez- Jiménez et al, realizada en México en el año 2014 que mide el autocuidado en enfermeras, señala que en su estudio de 265 profesionales el $60 \%$ presentaron prácticas de autocuidado inadecuadas, por lo tanto, estos resultados demuestran que los profesionales deben tomar medidas, ya que el ejercicio de su profesión contempla actividades que podrían poner en peligro la salud de los mismos. ${ }^{26}$

Dentro de las medidas de asociación entre las variables de autocuidado y percepción de riesgo se analizó que cuando los valores superan a 1 indican que es un factor de riesgo, mientras que los valores inferiores señalan un factor protector, en este caso el valor de 0,639 indica que las personas que tienen una baja percepción de riesgo es un factor protector para la baja capacidad de autocuidado, mientras una baja percepción de control de efectos negativos es un factor de riesgo para tener una baja capacidad de autocuidado porque, esta superior a la unidad 1,54. Al analizar los intervalos de confianza se pudo evidenciar que en ciertos momentos el factor protector y de riesgo 0,105 - 3,997 respectivamente demuestran que en ciertos casos puede existir un factor protector y un factor de riesgo.

En este estudio la relación entre percepción de riesgo laboral y autocuidado nos arroja un valor de 0,639 lo que indica que no existe asociación significativa entre las variables, sin embargo también se realizó una comparación de los riesgos percibidos por los distritos de salud, en el cual se evidencio que ambos presentan similares respuestas en "Trabajo con productos biológicos (medicinas, toxinas, vacunas, plasma etc)" y "Trabajo con elementos e instrumentación punzante o cortante (vidrio, agujas, punzones)" en donde el distrito 01D01 presenta un $45,2 \%$ y el distrito $01 \mathrm{D} 02$ un $54,8 \%$ y en lo que respecta a las ítems menos percibidos por ambos distritos están "Trabajo en sitios elevados" y "En mi puesto de trabajo utilizo con frecuencia aparatos de elevación ( grúas, ascensores, ect...).
Para confirmar lo antes mencionado se aplicó una prueba de U de Mann Whitney en la cual se evidencio que no existe diferencia en el número de riesgos percibidos por los distritos de salud (p 0,116). Los resultados de este estudio abren las puertas a nuevas investigaciones y a un mayor desarrollo y profundización del tema para así lograr una cultura de investigación en este nivel de la salud.

\section{Conclusiones}

En este estudio no se evidenció relación entre la percepción de riesgo laboral y autocuidado en los profesionales de enfermería que laboran en el primer nivel de atención en los Distritos 01D01 y 01D02, los profesionales que aplicaron sus conocimientos al responder las encuestas manifestaron que presentan una mediana percepción de riesgo. De la muestra obtenida se pudo evidenciar que las enfermeras y enfermeros se encuentran más expuestos a los riesgos de origen biológico en sus actividades diarias puesto que laboran constantemente con instrumentación corto punzante y se mantienen en contacto con varios microorganismos, los profesionales de los distritos 01D01 Y 01D02 de salud no presentaron una diferencia significativa ante la percepción de riesgo laboral.

\section{Fuente de Financiamiento}

Este estudio es autofinanciado.

\section{Conflicto de Intereses}

No existen conflictos personales, profesionales, financieroso de otro tipo.

\section{Consentimiento Informado}

Los autores cuentan con el consentimiento informado de los pacientes para la investigación, la publicación del caso y sus imágenes.

\section{Referencias Bibliográficas}

1. Barba E, Fernández S, Morales N, Rodriguez Nardelli AL, Bueno C, Giordano S. Salud Y Seguridad.2014;1-180.Disponible en: https://www.ilo. org/wcmsp5/groups/public/@americas/@ro-lima/@ilobuenos_aires/documents/publication/wcms_248685.pdf

2. Organización Mundial de la Salud. Protección de la salud de los trabajadores [Internet]. 30 de noviembre. 2017 [citado 23 de julio de 2019]. Disponible en: https://www.who.int/es/news-room/fact-sheets/detail/ protecting-workers $\backslash$ T1 $\backslash$ textquoteright-health

3. Organización Panamericana de la Salud. Salud de los trabajadores: Recursos [Internet]. 14 de abril. 2017 [citado 23 de julio de 2019]. Disponible en:

4. Espinoza Benavente MC, Espinoza Venegas M. Percepción De Riesgo Laboral Y Autocuidado En Enfermeros Atención Primaria De Una Comuna Del BioBío. Horiz Enferm [Internet]. 2018;29(1):6-17. Disponible en: http://publicaciones.horizonteenfermeria.uc.cl/ index.php/rhe/article/view/144 
5. Manzano MJR, Toapanta M, Andino X, Rueda ML. Enfermedades ocupacionales del personal de enfermería por desempeño de labores en turnos rotativos y la importancia de los protocolos ergonómicos de prevención. 1 Rev Científica Cienc y Tecnol [Internet]. 2018;1(18):116. Disponible en: http://181.39.139.66/revista/index.php/ cienciaytecnologia/article/view/336

6. Luengo Martínez C, Sanhueza O. Condiciones de trabajo y su relación con la calidad del cuidado y salud del profesional de enfermería. Med Se-17. gur Trab (Madr) [Internet]. 2016;62(245):368-80. Disponible en: http://scielo.isciii.es/scielo.php?script=sci_ arttext\&pid=S0465-546X2016000500008

7. Álvarez Heredia F, Conti Parra L, Valderrama Mantilla F, 18. Moreno Vargas O, Jiménez Barbosa I. Salud Ocupacional. En: Eco Ediciones, editor. Salud Ocupacional. 1ra ed. Bogotá; 2015. p. 15-20.

8. Arcanjo RVG, Chistovam BP, Braga ALDS, Silvino ZR. Gerenciamento dos riscos ocupacionais da 19. enfermagem na atenção básica: estudo exploratório descritivo / Management of occupational risks of nursing in primary health care: a descriptive exploratory 20. study. Rev Pesqui Cuid é Fundam Online [Internet]. 2018;10(2):351. Disponible en: https://www.researchgate. net/publication/324229152_Gerenciamento_dos_riscos_ ocupacionais_da_enfermagem_na_atencao_basica_ estudo_exploratorio_descritivo_Management_of_ occupational_risks_of_nursing_in_primary_health_ care_a_descriptive_exploratory_study

9. Zurro Martin A, Perez Cano J. Atención Primaria De Sa- 22 lud. Rev Fac Cienc Méd Enero-Junio [Internet]. 2015;(4). Disponible en: http://www.bvs.hn/RFCM/pdf/2015/pdf/ RFCMVol12-1-2015-2.pdf

10. Ministerio de Salud Pública. Manual del Modelo de Atencion Integral de Salud - MAIS. Msp. Ecuador; 2018. 81-84 p.

11. Ministerio de Salud Pública. Tipología para homologar Establecimientos de Salud por niveles. 2015;18. Disponi- 23. ble en: http://instituciones.msp.gob.ec/cz6/images/lotaip/ Enero2015/Acuerdo\%20Ministerial\%205212.pdf

12. Castillo ZR, Castillo ZR, Méndez PRC, Quezada LMT, Quezada CET, Fonseca RSS. Cumplimiento de las medidas de bioseguridad en la unidad quirúrgica de cirugía ambulatoria. Arch Médico Camagüey [Internet]. 5 de octubre de 2018 [citado 20 de noviembre de 2019];22(5):605-14. Disponible en: http://revistaamc.sld. 24 cu/index.php/amc/article/view/5704

13. Diaz-Tamayo AM, Vivas M MC. Riesgo biológico y prácticas de bioseguridad en docencia. Rev Fac Nac Salud Pública. 2015;34(1).

14. Colas Soria L, Iglesia Biot C, López Gonzáles D, Sayú Durand L. Aspectos sobre las medidas de bioseguridad del personal de Enfermería en servicios de hemodiálisis. Rev Inf Científica. 2014;83(1):144-52.

15. Ministerio de Salud Pública. ESTRATEGIA NACIONAL 25. De Pires DEP, Machado RR, Soratto J, Scherer DE INMUNIZACIONES [Internet]. Ecuador; 2019 [citado 21 de noviembre de 2019]. Disponible en: https://www.salud.gob.ec/wp-content/uploads/2019/04/ Esquema_de_vacunacion_MSP_2019-18-02-2019.pdf

6. Gámez de la Hoz J, Padilla Fortes A. Occupational risks identification at primary care through the workers communication. Rev la Asoc Esp Espec en Med del Trab [Internet]. 2017;26(1):22-30. Disponible en: http://scielo.isciii.es/pdf/medtra/v26n1/11326255-medtra-26-01-00022.pdf

7. Rivadeneira Silva R. Defensoría del Pueblo Resolución Ecuador [Internet]. 2017. Disponible en: https://www.dpe.gob.ec/lotaip/2017/pdfagosto/ JURIDICO/a3/RESOLUCION_054-2017.pdf

. Naranjo Hernández Y, Concepción Pacheco JA, Rodríguez Larreynaga M. Gaceta Médica Espirituana. Gac Médica Espirituana [Internet]. 2017;19(3):89-100. Disponible en: http://scielo.sld.cu/scielo.php?script=sci_arttext\&pid= S1608-89212017000300009\&lng=es\&nrm=iso\&tlng=es . Silvela F. Desarrollo de la enfermería-Teorías. En: Cañizares Artes Gráficas, editor. Manual CTO de Enfermería. 6ta ed. Madrid; 2014. p. 1453-6.

0. Prado L, Gonzales M, Paz N, Romero K, Romero Borges K. La teoría déficit de autocuidado Dorothea Orem punto de partida para calidad en la atención. Rev Med Electron [Internet]. 2014;36:835-45. Disponible en: http://scielo.sld.cu/scielo.php?script=sci_arttext\& pid=S1684-18242014000600004

21. Hernández MR. Autocuidado y promoción de la salud en el ámbito laboral. Rev Salud Bosque. 2016;5(2):79.

Universidad de Santander (Bucaramanga CP de E, Cañón-Montañez W. Revista Cuidarte. Rev Cuid [Internet]. 2015;6(1):879-81.Disponible en: https://www.researchgate.net/publication/331217681_ Agencia_de_autocuidado_conocimientos_actitudes_ y_practicas_de_riesgo_biologico_en_estudiantes_de_ enfermeria?fbclid=IwAR2TrhPydEoLHeDqs89kCFRnXqs4HKLee_xneM9MANrWHmJhriO1Vg_q30

3. Loro MM, Zeitoune RCG. Collective strategy for facing occupational risks of a nursing team TT - Estratégia coletiva de enfrentamento dos riscos ocupacionais de uma equipe de enfermagem TT - Estrategia colectiva de enfrentamiento de los riesgos ocupacionales del equipo de enfe. Rev Esc Enferm USP [Internet]. 2017;51:e03205e03205. Disponible en: http://www.scielo.br/scielo.php? script=sci_arttext\&pid=S0080-62342017000100402

. Vieira Girão Arcanjo R, Pompeu Christovam B, Dantas de Oliveira Souza NV, Silvino ZR, da Costa TF. Conocimientos y prácticas de los trabajadores de enfermería sobre riesgos laborales en la atención primaria de salud: un estudio de intervención TT - Knowledge and practices of nursing workers on occupational risks in primary health care: an intervention. Enfermería Glob [Internet]. 2018;17(51):200-37. Disponible en: http://scielo.isciii.es/ pdf/eg/v17n51/1695-6141-eg-17-51-200.pdf M dos A, Gonçalves ASR, Trindade LL. Cargas 
de trabalho da enfermagem na saúde da família: Implicações no acesso universal. Rev Lat Am

Enfermagem [Internet]. 2016;24. Disponible en: http://www.scielo.br/scielo.php?script=sci_arttext\& pid=S0104-11692016000100313\&lng=en\&tlng=en

26. Sánchez-Jiménez B, Sámano R, Chinchilla-Ochoa D, Rodríguez-Ventura AL. Autocuidado en enfermeras y su asociación con su estado emocional. Salud Publica Mex [Internet]. 2014;56(3):2356. Disponible en: http://www.scielo.org.mx/scielo.php?script=sci_ arttext\&pid=S003636342014000300001

Recibido: 10 de octubre de 2020

Aceptado: 25 de octubre de 2020

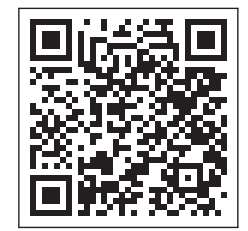


